\title{
Galerkin Strategy for Level Set Shape Analysis: Application to Geodesic Tube
}

\author{
Louis Blanchard ${ }^{1}$ and Jean-Paul Zolésio ${ }^{2}$ \\ 1 INRIA, Sophia Antipolis, France \\ louis.blanchardasophia.inria.fr \\ 2 CNRS and INRIA, 2004 route des Lucioles, BP 93, \\ 06902 Sophia Antipolis Cedex, France \\ jean-paul.zolesio@sophia.inria.fr
}

\begin{abstract}
In this paper, we consider the geodesic tube characterization using a Galerkin-Level Set strategy. The first section is devoted to the analysis of a geodesic tube construction between two sets through the definition of the shape metric. In the second section, we define the Galerkin-Level Set strategy in shape analysis. This new variational formulation associated to a Hilbert space metric for shape identification problem consists in parameterizing the level set function in a finite dimensional subspace spanned by linear independent functions. Consequently, this method is more focused on topological changes than on high accuracy for the boundary evaluation as in a traditional level set formulation. In the third section, we use the Galerkin-Level Set formulation applied to a geodesic tube construction between two sets, through the calculus of the shape derivative of the normal speed. Finally, this geodesic tube construction is validated by a numerical experiment.
\end{abstract}

\section{Tube Formulation Using Moving Domain}

In this section, we briefly recall the concept of connecting tube, introduced in [6]. Let us consider $\mathbb{D}$ as a bounded universe in $\mathbb{R}^{n}$ and two open sets domains $\Omega_{0}, \Omega_{1} \subset \mathbb{D}$. We denote the initial domain by $\Omega_{0}$ and the final domain by $\Omega_{1}$, and consider the tube connecting $\Omega_{0}$ with $\Omega_{1}$ defined by the $n+1$ dimensional graph of an $n$-dimensional moving domain: see Fig. 1 Consequently, considering the time interval $I=[0,1]$, we define the tube evolution $Q$ by product space, using the cylinder $I \times \Omega$ as follows:

$$
Q=\bigcup_{0 \leq t \leq 1}\{t\} \times \Omega_{t}
$$

Moreover, we denote by $\Sigma$ the lateral boundary of the tube, defined by the following expression: $\Sigma=\bigcup_{0 \leq t \leq 1}\{t\} \times \Gamma_{t}$, where $\Gamma_{t}$ denotes the boundary of $\Omega_{t}$. The characteristic function of the tube is defined by $\zeta(t, x) \stackrel{\text { def }}{=} \chi_{\Omega_{t}}(x)$ and verifies $\zeta^{2}=\zeta$. Following [4]5], the set of connecting tubes between $\Omega_{0}$ and $\Omega_{1}$ is defined by:

$$
\mathscr{T}\left(\Omega_{0}, \Omega_{1}\right)=\left\{\zeta \in L^{\infty}(I \times \mathbb{D}) \text { and piecewise } C^{1},\left[\begin{array}{l}
\zeta(0)=\chi_{\Omega_{0}} \\
\zeta(1)=\chi_{\Omega_{1}}
\end{array}\right\}\right.
$$




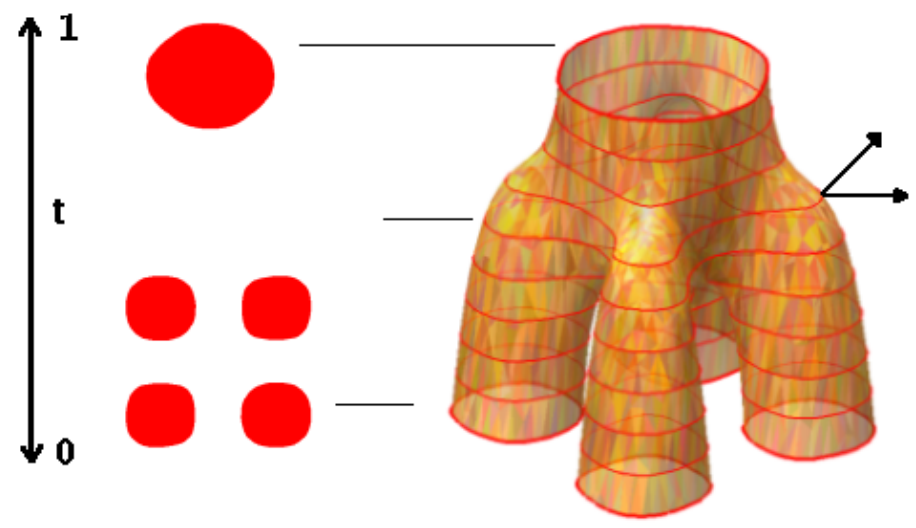

Fig. 1. Continuous tube between $\Omega_{0}$ and $\Omega_{1}$

The outgoing unitary normal vector field on the lateral boundary of the tube $\Sigma$ is defined by

$$
v(t, x)=\frac{1}{\sqrt{1+v(t, x)^{2}}}\left(\begin{array}{l}
-v(t, x) \\
\mathbf{n}(t, x)
\end{array}\right)
$$

where $\mathbf{n}(t, x)$ is the normal field to $\Gamma_{t}$ and $v(t, x)$ is an intrinsic geometric entity called the normal speed of the boundary $\Gamma_{t}$.

Definition 1. In order to characterize the minimal tube path between $\Omega_{0}$ and $\Omega_{1}$, we introduce the function:

$$
d\left(\Omega_{0}, \Omega_{1}\right)=\inf _{\zeta \in \mathscr{T}\left(\Omega_{0}, \Omega_{1}\right)} \int_{0}^{1} \int_{\Gamma_{t}}|v(t, x)| d \Gamma(x) d t
$$

Lemma 1. The function $d\left(\Omega_{0}, \Omega_{1}\right)$ is a metric.

Proof. We have to prove that the function $d\left(\Omega_{0}, \Omega_{1}\right)$ satisfies:

I. (Identity of indiscernibles) $d\left(\Omega_{0}, \Omega_{1}\right)=0 \Leftrightarrow \Omega_{0}=\Omega_{1}$.

- If $\Omega_{0}=\Omega_{1}$ then $v=0$ and $d\left(\Omega_{0}, \Omega_{1}\right)=0$.

- If $d\left(\Omega_{0}, \Omega_{1}\right)=0$ that implies $\forall t \in[0,1], v(t,)=$.0 and the time space normal (3) is $v(t,)=.(0, \mathbf{n}(t,)$.$) . Then the tube is a cylinder and the domain \Omega_{t}$ does not depend on time, consequently $\Omega_{0}=\Omega_{1}$.

II. (Symmetry) $d\left(\Omega_{0}, \Omega_{1}\right)=d\left(\Omega_{1}, \Omega_{0}\right)$.

- If we consider the backward tube $\hat{\zeta}(t)=\zeta(1-t) \in \mathscr{T}\left(\Omega_{1}, \Omega_{0}\right)$, that implies $\hat{v}(t,)=.-v(1-t,$.$) , and consequently d\left(\Omega_{0}, \Omega_{1}\right)=d\left(\Omega_{1}, \Omega_{0}\right)$.

III. (Triangle inequality) $d\left(\Omega_{0}, \Omega_{2}\right) \leq d\left(\Omega_{0}, \Omega_{1}\right)+d\left(\Omega_{1}, \Omega_{2}\right)$.

- We consider three open sets domains in $\mathbb{D}: \Omega_{i} \forall i \in[0,2]$. We denote by $\zeta_{1} \in$ $\mathscr{T}\left(\Omega_{0}, \Omega_{1}\right)$ the tube connecting $\Omega_{0}$ to $\Omega_{1}$, and by $\zeta_{2} \in \mathscr{T}\left(\Omega_{1}, \Omega_{2}\right)$ the tube 
connecting $\Omega_{1}$ to $\Omega_{2}$. Let us consider the piecewise $C^{1}$ tube defined, through its characteristic function $\hat{\zeta}$ as follows:

$$
\hat{\zeta}(t, x)=\left\{\begin{array}{ccc}
\zeta_{1}(2 t, x) & \text { if } & 0 \leq t \leq \frac{1}{2} \\
\zeta_{2}(2 t-1, x) & \text { if } & \frac{1}{2} \leq t \leq 1
\end{array}\right.
$$

Consequently, the normal speed on the boundary $\Gamma_{t}$ is given by:

$$
\hat{v}(t, x)=\left\{\begin{array}{ccc}
2 v_{1}(2 t, x) & \text { if } & 0 \leq t \leq \frac{1}{2} \\
2 v_{2}(2 t-1, x) & \text { if } & \frac{1}{2} \leq t \leq 1
\end{array}\right.
$$

Now by construction $\hat{\zeta} \in \mathscr{T}\left(\Omega_{0}, \Omega_{2}\right)$ is a tube connecting $\Omega_{0}$ to $\Omega_{2}$ and we get:

$$
\begin{aligned}
d\left(\Omega_{0}, \Omega_{2}\right) & \leq \int_{0}^{\frac{1}{2}} \int_{\Gamma_{t}}|\hat{v}(t, x)| d \Gamma(x) d t+\int_{\frac{1}{2}}^{1} \int_{\Gamma_{t}}|\hat{v}(t, x)| d \Gamma(x) d t \\
& \leq \int_{0}^{\frac{1}{2}} \int_{\Gamma_{t}}\left|2 v_{1}(2 t, x)\right| d \Gamma(x) d t+\int_{\frac{1}{2}}^{1} \int_{\Gamma_{t}}\left|2 v_{2}(2 t-1, x)\right| d \Gamma(x) d t \\
& \leq \int_{0}^{1} \int_{\Gamma_{t}}\left|v_{1}(r, x)\right| d \Gamma(x) d r+\int_{0}^{1} \int_{\Gamma_{t}}\left|v_{2}(u, x)\right| d \Gamma(x) d u
\end{aligned}
$$

and as $v_{1}$ (resp. $v_{2}$ ) is the infimum in the definition of $d\left(\Omega_{0}, \Omega_{1}\right)$ (resp. $\left.d\left(\Omega_{1}, \Omega_{2}\right)\right)$ up to $\varepsilon>0$, then $\forall \varepsilon \in \mathbb{R}_{+}^{*}$ we get:

$$
d\left(\Omega_{0}, \Omega_{2}\right) \leq d\left(\Omega_{0}, \Omega_{1}\right)+d\left(\Omega_{1}, \Omega_{2}\right)+2 \varepsilon
$$

\subsection{Tube Formulation Using a Level Set Method}

In this paper, we use a level set parameterization for the domain evolution. In this method the moving domain $\Omega_{t}$ is defined by the set of points in $\mathbb{D}$ for which the level set function $\Phi$ is positive:

$$
\Omega_{t}=\{x \in \mathbb{D} \mid \Phi(t, x)>0\}
$$

We denote by $\Phi_{0}$ the level set function of the domain $\Omega_{0}$, and by $\Phi_{1}$ the level set function of the domain $\Omega_{1}$ :

$$
\Omega_{0}=\left\{x \in \mathbb{D} \mid \Phi_{0}(x)>0\right\}, \Omega_{1}=\left\{x \in \mathbb{D} \mid \Phi_{1}(x)>0\right\}
$$

Using the level set formulation, the set of connecting tubes between the initial domain $\Omega_{0}$ and the final domain $\Omega_{1}$ becomes:

$$
\mathscr{T}_{L S}\left(\Omega_{0}, \Omega_{1}\right)=\left\{\begin{array}{l}
\Phi(t, x) \in L^{1}\left(I, C^{0}(\overline{\mathbb{D}})\right) \\
\chi_{\Omega_{t}} \in C^{0}\left(\bar{I}, L^{1}(\mathbb{D})\right) \\
\Phi \text { piecewise } C^{0}
\end{array},\left[\begin{array}{l}
\Phi(0, x)=\Phi_{0}(x) \\
\Phi(1, x)=\Phi_{1}(x)
\end{array}\right\}\right.
$$


We consider a decomposition of the time interval $I$ into a finite number of time intervals in which the level set function $\Phi$ is continuous. Therefore $\Phi$ is piecewise $C^{0}$, which means that there exists an integer $N$ and an increasing sequence: $\left(t_{0}=0<t_{1}<\cdots<\right.$ $\left.t_{N}=1\right)$ with a decomposition of the time interval as follows: $I=\bigcup_{1 \leq k \leq N} \bar{I}_{k}$ where $I_{k}=$ ]$t_{k}, t_{k+1}[$, such that:

$$
\forall k \in[1, N],\left.\Phi(t, .)\right|_{I_{k}} \in C^{0}\left(I_{k}\right)
$$

Definition 2. The metric d defined by the equation (4) can be expressed, in term of the level set function $\Phi$ as follows:

$$
d\left(\Omega_{0}, \Omega_{1}\right)=\inf _{\Phi \in \mathscr{T}_{L S}\left(\Omega_{0}, \Omega_{1}\right)} \int_{0}^{1} \int_{\Gamma_{t}=\Phi^{-1}(0)} \frac{\left|\partial_{t} \Phi(t, x)\right|}{\|\nabla \Phi(t, x)\|} d \Gamma(x) d t
$$

Indeed, using the level set formulation we have the relations:

$$
\mathbf{n}(t, x)=\frac{-\nabla \Phi(t, x)}{\|\nabla \Phi(t, x)\|}, \quad \mathbf{V}(t, x)=-\partial_{t} \Phi(t, x) \frac{\nabla \Phi(t, x)}{\|\nabla \Phi(t, x)\|}
$$

Then the normal speed of the boundary $\Gamma_{t}$ turns into:

$$
v(t, x)=\langle\mathbf{V}(t, x), \mathbf{n}(t, x)\rangle_{\mathbb{R}^{n}}=\frac{\partial_{t} \Phi(t, x)}{\|\nabla \Phi(t, x)\|}
$$

where $\langle., .\rangle_{\mathbb{R}^{n}}$ denotes the inner product in $\mathbb{R}^{n}$.

Assumption 1. The function $d\left(\Omega_{0}, \Omega_{1}\right)$ expressed in term of the level set function $\Phi$, is also a metric.

\subsection{Tube Formulation Using the Federer Theorem}

In this section, we consider the tube formulation through the level set method described previously, and we consider an approximation of the metric $d$ using the Federer measure decomposition theorem.

Theorem 1 (Federer measure decomposition). Let us consider a functional $F \in L^{1}(\mathbb{D})$, and $\forall h>0$ the domain

$$
U_{h}\left(\Gamma_{t}\right)=\{x \in \mathbb{D} \mid\|\Phi(t, x)\|<h\}
$$

Then we have:

$$
\int_{U_{h}(\Gamma)} F(x) d x=\int_{-h}^{+h}\left(\int_{\Phi^{-1}(z)} \frac{F(x)}{\left\|\nabla_{x} \Phi(x)\right\|} d \Gamma(x)\right) d z
$$

Corollary 1. Assuming the mapping:

$$
z \in[-h,+h] \rightarrow \int_{\Phi^{-1}(z)} \frac{F(x)}{\left\|\nabla_{x} \Phi(x)\right\|} d \Gamma
$$

to be continuous, we obtain:

$$
\int_{\Gamma} \frac{F(x)}{\left\|\nabla_{x} \Phi(x)\right\|} d \Gamma(x)=\frac{1}{2 h} \int_{U_{h}(\Gamma)} F(x) d x+o(1), h \rightarrow 0
$$


Definition 3. Using the Federer measure decomposition theorem and according to the previous corollary, we consider an approximation of the metric $d$ denoted by $d_{h}$ and defined as follows:

$$
d_{h}\left(\Omega_{0}, \Omega_{1}\right)=\inf _{\Phi \in \mathscr{T}_{L S}\left(\Omega_{0}, \Omega_{1}\right)} \int_{0}^{1} \frac{1}{2 h} \int_{U_{h}\left(\Gamma_{t}\right)}\left|\partial_{t} \Phi(t, x)\right| d x d t
$$

Lemma 2. The approximation of the metric $d\left(\Omega_{0}, \Omega_{1}\right)$, denoted $d_{h}\left(\Omega_{0}, \Omega_{1}\right)$ is also a metric.

Proof. We have to prove that the function $d_{h}\left(\Omega_{0}, \Omega_{1}\right)$ satisfies:

I. (Identity of indiscernibles) $d_{h}\left(\Omega_{0}, \Omega_{1}\right)=0 \Leftrightarrow \Omega_{0}=\Omega_{1}$.

- If $\Omega_{0}=\Omega_{1}$ then $\partial_{t} \Phi=0$ and $d_{h}\left(\Omega_{0}, \Omega_{1}\right)=0$.

- If $d_{h}\left(\Omega_{0}, \Omega_{1}\right)=0$ that implies $\forall t \in[0,1], \partial_{t} \Phi(t,)=$.0 in $\mathscr{D}_{h}=\bigcup_{0 \leq t \leq 1}\{t\} \times U_{h}\left(\Gamma_{t}\right)$ and that implies $\Phi=\Phi(x) \in \mathscr{D}_{h}$. Consequently, the boundary $\Gamma_{t} \stackrel{\text { def }}{=}\{x \in \mathbb{D} \mid \Phi(x)=0\}$ does not depend on time, and $\Omega_{0}=\Omega_{1}$.

II. (Symmetry) $d_{h}\left(\Omega_{0}, \Omega_{1}\right)=d_{h}\left(\Omega_{1}, \Omega_{0}\right)$.

- If we consider the backward tube $\hat{\Phi}(t,)=.\Phi(1-t,.) \in \mathscr{T}\left(\Omega_{1}, \Omega_{0}\right)$, that implies $\partial_{t} \hat{\Phi}(t,)=.-\partial_{t} \Phi(1-t,$.$) , and d_{h}\left(\Omega_{0}, \Omega_{1}\right)=d_{h}\left(\Omega_{1}, \Omega_{0}\right)$.

III. (Triangle inequality) $d_{h}\left(\Omega_{0}, \Omega_{2}\right) \leq d_{h}\left(\Omega_{0}, \Omega_{1}\right)+d_{h}\left(\Omega_{1}, \Omega_{2}\right)$.

- We assume three open sets domains in $\mathbb{D}: \Omega_{i} \forall i \in[0,2]$. We denote by $\Phi_{1} \in$ $\mathscr{T}_{L S}\left(\Omega_{0}, \Omega_{1}\right)$ the tube connecting $\Omega_{0}$ to $\Omega_{1}$, and by $\Phi_{2} \in \mathscr{T}_{L S}\left(\Omega_{1}, \Omega_{2}\right)$ the tube connecting $\Omega_{1}$ to $\Omega_{2}$. Let us consider the piecewise $C^{1}$ tube defined, through its level set function $\Phi$ as follows:

$$
\bar{\Phi}(t, x)=\left\{\begin{array}{ccc}
\Phi_{1}(2 t, x) & \text { if } & 0 \leq t \leq \frac{1}{2} \\
\Phi_{2}(2 t-1, x) & \text { if } & \frac{1}{2} \leq t \leq 1
\end{array}\right.
$$

Consequently, the time derivative of level set function $\Phi$ on the domain $U_{h}\left(\Gamma_{t}\right)$ is given by:

$$
\partial_{t} \bar{\Phi}(t, x)(t, x)=\left\{\begin{array}{ccc}
2 \partial_{t} \Phi_{1}(2 t, x) & \text { if } & 0 \leq t \leq \frac{1}{2} \\
2 \partial_{t} \Phi_{2}(2 t-1, x) & \text { if } & \frac{1}{2} \leq t \leq 1
\end{array}\right.
$$

Now by construction $\bar{\Phi} \in \mathscr{T}_{L S}\left(\Omega_{0}, \Omega_{2}\right)$ is a tube connecting $\Omega_{0}$ to $\Omega_{2}$ and we get:

$$
\begin{aligned}
& d_{h}\left(\Omega_{0}, \Omega_{2}\right) \leq \int_{0}^{\frac{1}{2}} \frac{1}{2 h} \int_{U_{h}\left(\Gamma_{t}\right)}\left|\partial_{t} \bar{\Phi}(t, x)\right| d x d t+\int_{\frac{1}{2}}^{1} \frac{1}{2 h} \int_{U_{h}\left(\Gamma_{t}\right)}\left|\partial_{t} \bar{\Phi}(t, x)\right| d x d t \\
& \leq \frac{1}{2 h}\left[\int_{0}^{\frac{1}{2}} \int_{U_{h}\left(\Gamma_{t}\right)}\left|2 \partial_{t} \Phi_{1}(2 t, x)\right| d x d t+\int_{\frac{1}{2}}^{1} \int_{U_{h}\left(\Gamma_{t}\right)}\left|2 \partial_{t} \Phi_{2}(2 t-1, x)\right| d x d t\right] \\
& \leq \int_{0}^{1} \frac{1}{2 h} \int_{U_{h}\left(\Gamma_{t}\right)}\left|\partial_{t} \Phi_{1}(r, x)\right| d x d r+\int_{0}^{1} \frac{1}{2 h} \int_{U_{h}\left(\Gamma_{t}\right)}\left|\partial_{t} \Phi_{2}(u, x)\right| d x d u
\end{aligned}
$$


and as $\Phi_{1}$ (resp. $\Phi_{2}$ ) is the infimum in the definition of $d_{h}\left(\Omega_{0}, \Omega_{1}\right)$ (resp. $\left.d_{h}\left(\Omega_{1}, \Omega_{2}\right)\right)$ up to $\varepsilon>0$. Then $\forall \varepsilon \in \mathbb{R}_{+}^{*}$, we get

$$
d_{h}\left(\Omega_{0}, \Omega_{2}\right) \leq d_{h}\left(\Omega_{0}, \Omega_{1}\right)+d_{h}\left(\Omega_{1}, \Omega_{2}\right)+2 \varepsilon
$$

\section{Shape Identification Problem}

We address the question concerning the shape identification of a given smooth domain. A commonly used approach in shape analysis consists in choosing a level set formulation for the evolution of moving domain. The main advantage of a level set formulation concerns the easy generation of topological changes during the evolution process.

\subsection{Shape Identification Using a Level Set Method}

Let us denote by $\Omega_{*} \in \mathbb{D}$ a smooth domain to identify and by $\chi_{\Omega_{*}}$, its characteristic function satisfying: $\chi_{\Omega_{*}} \in H^{s}(\mathbb{D}), 0<s<\frac{1}{2}$. Following [1], the evaluation of the distance between the given domain $\Omega_{*}$ and the moving domain $\Omega_{t}$ is made by the use of a metric associated to the Hilbert space $H^{s}$ denoted $\delta_{s}\left(\Omega, \Omega_{*}\right)$ and defined by:

$$
\begin{aligned}
\forall s \in] 0, \frac{1}{2}\left[, \quad \delta_{s}\left(\Omega, \Omega_{*}\right)\right. & =\left\|\chi_{\Omega}-\chi_{\Omega_{*}}\right\|_{H^{s}(\mathbb{D})} \\
& =\left\|\chi_{\Omega}-\chi_{\Omega_{*}}\right\|_{L^{2}(\mathbb{D})}+\left\|\chi_{\Omega}-\chi_{\Omega_{*}}\right\|_{s}
\end{aligned}
$$

where

$$
\left\|\chi_{\Omega}\right\|_{s}^{2}=\int_{\mathbb{D}} \int_{\mathbb{D}}\left|\chi_{\Omega}(x)-\chi_{\Omega}(y)\right|^{2} G(x, y) d x d y
$$

and where the kernel function defined by: $G(x, y)=|x-y|^{-(n+2 s)}$ is singular on the diagonal $\Delta=\{(x, x) \subset \mathbb{D} \times \mathbb{D}, x \in \mathbb{D}\}$.

\subsubsection{Shape Analysis via the Speed Method}

Finally, we use the concept of speed method from shape analysis [3] to compute the shape derivative of the metric $\delta_{s}\left(\Omega, \Omega_{*}\right)$ which corresponds to a gradient direction for the underling shape optimization problem:

$$
\min _{\Omega \in \mathbb{D}} \delta_{S}\left(\Omega, \Omega_{*}\right)
$$

Definition 4. Let us consider an open set domain $\Omega$ where $\Gamma=\partial \Omega$ is of class $C^{1}$. We define the eulerian derivative of the functional $J$ in the direction of a perturbation vector field $\mathbf{W} \in C_{0}^{1}(\mathbb{D} ; \mathbb{D})$ by

$$
d J\left(\Gamma_{t}, \mathbf{W}\right)=\left.\frac{\partial J(\Gamma(t+\varepsilon))}{\partial \varepsilon}\right|_{\varepsilon=0}
$$

Lemma 3. The funtional $\delta_{s}\left(\Omega, \Omega_{*}\right)$ is shape derivative for perturbation vector fields $\mathbf{V} \in C_{0}^{1}(\mathbb{D}, \mathbb{D})$, and expressed as follows: 


$$
d \delta_{S}\left(\left(\Omega, \Omega_{*}\right) ; \mathbf{V}\right)=\int_{\Gamma} F(x)\langle\mathbf{V}(0, x), \mathbf{n}(x)\rangle_{\mathbb{R}^{n}} d \Gamma(x)
$$

where $d \Gamma$ is the arclength measure on $\Gamma$ and where:

$$
F(x)=\frac{1-2 \chi_{\Omega_{*}}(x)}{2\left\|\chi_{\Omega}-\chi_{\Omega_{*}}\right\|_{L^{2}(\mathbb{D})}}+\frac{\int_{\mathbb{D}}\left[1-2 \chi_{\Omega_{t}}(y)+2\left[\chi_{\Omega_{*}}(y)-\chi_{\Omega_{*}}(x)\right]\right] G(x, y) d y}{\left\|\chi_{\Omega}-\chi_{\Omega_{*}}\right\|_{s}}
$$

Proof. See [1].

\subsection{Shape Identification Using a Galerkin-Level Set Strategy}

Generally, the parameterization of the level set function $\Phi$ is done by the oriented distance function denoted $b_{\Omega_{t}}$, see [2]3] for references:

$$
\Phi(t, x)=-b_{\Omega_{t}}(x)
$$

where $b_{\Omega}(x)$ is also called signed distance function and is defined as follows:

$$
b_{\Omega}(x)=d_{\Omega}(x)-d_{\complement \Omega}(x) \quad \text { with } \quad d_{A}(x)=\inf _{y \in A}|y-x|
$$

The choice of the oriented distance function for the parameterization of the level set function can be necessary for having a high accuracy of the boundary approximation. However, the choice of the oriented distance function implies an expansive computational cost owing to the complexity of its evaluation and imposes a reinitialization during the evolution process. Consequently, according to the fact that in this paper we focus on topological changes without considering the approximation of the boundary as an essential point, we use a new approach called Galerkin-Level Set method.

\subsubsection{Galerkin-Level Set Strategy}

The Galerkin-Level Set strategy consists in parameterizing the level set function in a finite dimensional subspace $\mathscr{E}$, spanned by linear independent functions defined over $\mathbb{D}$ $: \mathscr{E}=\left\{E_{1}, \ldots, E_{m}\right\}$. We denote by $\Lambda(t)=\left(\lambda_{1}(t), \ldots, \lambda_{m}(t)\right)$ the parameter vector of the Galerkin decomposition of $\Phi$ in the basis $\mathscr{E}$ :

$$
\Phi(t, x)=\sum_{k=1}^{m} \lambda_{k}(t) E_{k}(x)
$$

Consequently, using the Galerkin decomposition of the level set function, the parameterization of the moving domain $\Omega(t)$ is defined as follows:

$$
\Omega(t)=\left\{x \in \mathbb{D} \mid \Phi(t, x)=\sum_{k=1}^{m} \lambda_{k}(t) E_{k}(x)>0\right\}
$$




\subsubsection{Level Set Equation}

In a level set formulation, the moving domain evolves by advecting the level set function $\Phi$ following the flow of the shape gradient. Then, in a traditional level set formulation, the transport equation is a Partial Differential Equation (PDE) of Hamilton-Jacobi type:

$$
\left\{\begin{aligned}
\partial_{t} \Phi(t, x)+\rho F(x)\|\nabla \Phi(t, x)\| & =0, & & \rho>0 \\
\Phi(0, x) & =\Phi_{0}(x), & & (t, x) \in[0, \tau] \times \Omega_{t}
\end{aligned}\right.
$$

Remark 1. The main advantage of the Galerkin-Level Set method compared to the traditional level set formulation concerns the level set equation that turns, in the GalerkinLevel Set method, into a system of ordinary differential equations.

Lemma 4. Using the Galerkin-Level Set strategy (34), the level set equation turns into a system of $m$ ordinary differential equations:

$$
\left\{\begin{aligned}
\partial_{t} \Lambda(t)+\rho \mathscr{F}(t, x) & =0, & & \rho>0 \\
\Lambda(0) & =\Lambda_{0}, & & (t, x) \in[0, \tau] \times \Omega_{t}
\end{aligned}\right.
$$

where

$$
\mathscr{F}(t, x)=\left(\int_{\Gamma_{t}} \frac{F(x)}{\|\nabla \Phi(t, x)\|} E_{1}(x) d \Gamma(x), \ldots, \int_{\Gamma_{t}} \frac{F(x)}{\|\nabla \Phi(t, x)\|} E_{m}(x) d \Gamma(x)\right)
$$

Proof. According to the Galerkin-Level Set strategy, consisting in the decomposition of function $\Phi(33)$, the shape derivative of the functional $\delta_{s}\left(\Omega, \Omega_{*}\right)$ with respect to the vector of parameters $\Lambda(t)$ turns into:

$$
d \delta_{s}\left(\left(\Omega, \Omega_{*}\right) ; \mathbf{V}\right)=\sum_{k=1}^{m} \partial_{t} \lambda_{k}(t) \int_{\Gamma_{t}} F(x) \frac{E_{k}(x)}{\|\nabla \Phi(t, x)\|} d \Gamma(x)
$$

where only the vector of parameters $\Lambda(t)$ depends on time. A sufficient condition to decrease the shape gradient is to choose:

$$
\forall k \in[1, m], \forall \rho \in \mathbb{R}_{+}^{*}, \quad \partial_{t} \lambda_{k}(t)=-\rho \int_{\Gamma_{t}} F(x) \frac{E_{k}(x)}{\|\nabla \Phi(t, x)\|} d \Gamma(x)
$$

Finally, considering the level set equation we obtain a system of $m$ ordinary differential equations (36).

Corollary 2. Substituting the approximation of the boundary integral calculus from the equation (19), into the system of $m$ ordinary differential equations (37), we obtain an approximation of the vector $\mathscr{F}$ defined as follows:

$$
\tilde{\mathscr{F}}(t, x)=\left(\frac{1}{2 h} \int_{U_{h}\left(\Gamma_{t}\right)} F(x) E_{1}(x) d x, \ldots, \frac{1}{2 h} \int_{U_{h}\left(\Gamma_{t}\right)} F(x) E_{m}(x) d x\right)
$$

Note that in this new formulation the main advantage is that the denominator term $\|\nabla \Phi(x)\|$ has been eliminated. 
From now, we use the previous corollary for the level set equation and we consider the following algorithm.

\section{Algorithm 1}

I. Initialization: Choose an initial vector of parameters $\Lambda_{0}=\left(\lambda_{1}^{0}, \ldots, \lambda_{m}^{0}\right)$. Initialize the level set function $\Phi_{0}(x)=\sum_{l=1}^{m} \lambda_{l}^{0} E_{l}(x)$. Set $k=0$.

II. Shape gradient direction: Find the tubular neighborhood $U_{h}\left(\Gamma_{t_{k}}\right)$ of the zero level set $\Gamma_{t_{k}}$ of the actual level set function $\Phi\left(t_{k}, x\right)$. Compute $\tilde{\mathscr{F}}\left(t_{k}, x\right)$ from the equation (40).

III. Update: Perform a time step in the level set equation 36 to update $\Lambda\left(t_{k}\right)$. Let $\Lambda\left(t_{k+1}\right)$ denote this update: $\Lambda\left(t_{k+1}\right)=\Lambda\left(t_{k}\right)-\rho \tilde{\mathscr{F}}\left(t_{k}, x\right), \rho>0$. Update the function $\Phi\left(t_{k+1}, x\right)=\sum_{l=1}^{m} \lambda_{l}\left(t_{k+1}\right) E_{l}(x)$. Set $k=k+1$ and go to 2 .

\subsection{Numerical Experiment}

We present a numerical experiment based on the algorithm 1 for a 3D shape identification problem using the Galerkin-Level Set method described in the previous section. In this numerical experiment, the given domain $\Omega_{*}$ to identify is the gray matter of a human brain. We consider a Galerkin-Level Set expansion of the level set function $\Phi$ in Fourier series of dimension $m=25^{3}$; note that in this $3 \mathrm{D}$ case the level set function $\Phi$ is in $\mathbb{R}^{4}$. We start with a smooth initial domain $\Omega_{t=0}$ corresponding to the lower frequency of the Fourier series: see left-hand picture in Fig. 2. The algorithm detects the contour of the human brain after only 8 iterations.

\section{Geodesic Tube Formulation Using Moving Domain}

\subsection{Tube Formulation Using a Galerkin Strategy}

The tube path between $\Omega_{0}$ and $\Omega_{1}$ is made by a Galerkin-Level Set approach. The moving domain $\Omega_{t}$ of the tube evolution defined by the equation 10 is parameterized by the Galerkin-Level Set formulation and defined as follows:

$$
\Omega(t)=\left\{x \in \mathbb{D} \mid \Phi(t, x)=\sum_{k=1}^{m} \lambda_{k}(t) E_{k}(x)>0\right\}
$$

where $\Lambda(t)=\left(\lambda_{1}(t), \ldots, \lambda_{m}(t)\right) \in \mathbb{R}^{m}$ is the vector of parameters in the Galerkin expansion of the level set function. The first step consists in identifying the initial domain $\Omega_{0}$ and the final domain $\Omega_{1}$ through the research of the parameters $\Lambda_{0}=\left(\lambda_{1}^{0}, \ldots, \lambda_{m}^{0}\right) \in \mathbb{R}^{m}$ and $\Lambda_{1}=\left(\lambda_{1}^{1}, \ldots, \lambda_{m}^{1}\right) \in \mathbb{R}^{m}$ which satisfy the equations:

$$
\Phi_{0}(x)=\sum_{k=1}^{m} \lambda_{k}^{0} E_{k}(x), \quad \Phi_{1}(x)=\sum_{k=1}^{m} \lambda_{k}^{1} E_{k}(x)
$$

Thus, the feasible set of connecting tubes between $\Omega_{0}$ and $\Omega_{1}$ through the GalerkinLevel Set formulation turns into:

$$
\mathscr{T}_{\Lambda}\left(\Omega_{0}, \Omega_{1}\right)=\left\{\Lambda(t) \in\left(L^{2}(I)\right)^{m},\left[\begin{array}{l}
\Lambda(0)=\Lambda_{0} \\
\Lambda(1)=\Lambda_{1}
\end{array}\right\}\right.
$$



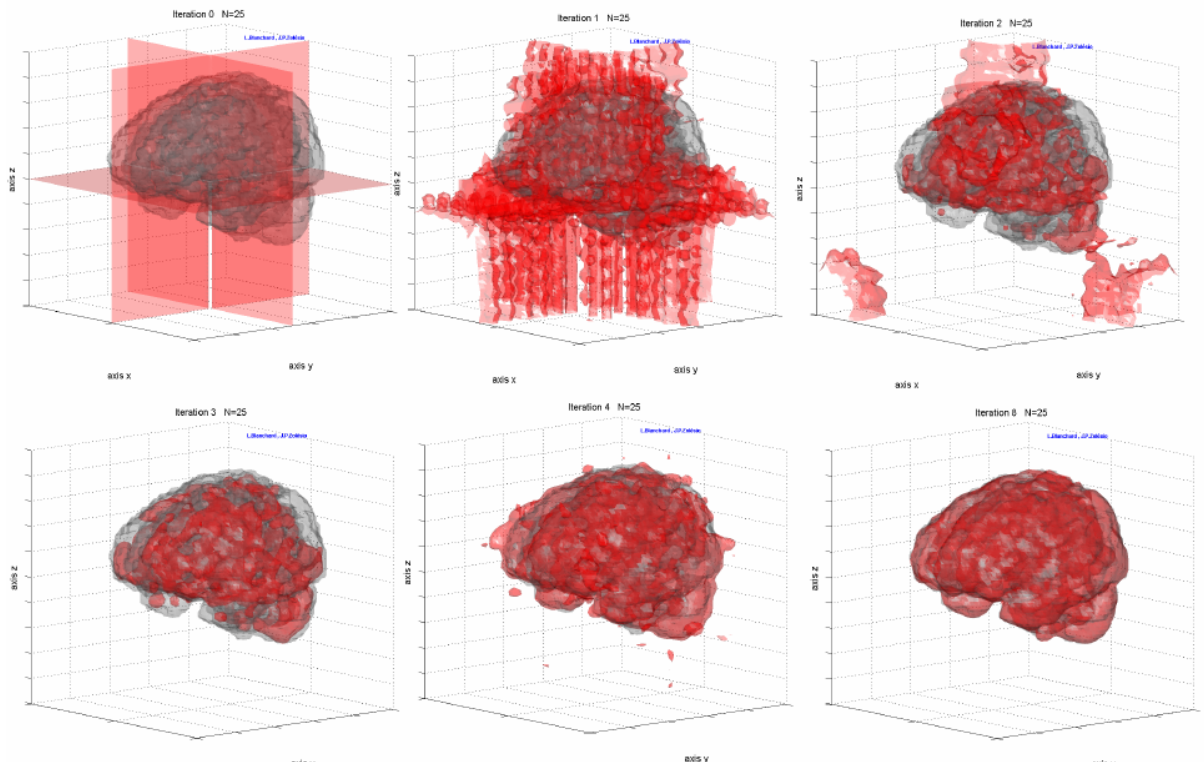

$\operatorname{sis} x$

Fig. 2. Shape identification of gray matter of human brain using a Galerkin-Level Set method

Remark 2. The feasible set of connecting tubes between $\Omega_{0}$ and $\Omega_{1}$ is not empty. Indeed, if we consider the vector of parameters $\Lambda(t)$ as a convex combination of $\Lambda_{0}$ and $\Lambda_{1}: \Lambda(t)=\Lambda_{1} t+\Lambda_{0}(1-t)$, we have $\Lambda(t) \in \mathscr{T}_{\Lambda}\left(\Omega_{0}, \Omega_{1}\right)$. Moreover, the parameters $\Lambda(t)$ defined as a convex combination of $\Lambda_{0}$ and $\Lambda_{1}$ generate an admissible tube that we use for the initialization during the tube optimization process.

\subsection{Geodesic Tube Construction between Two Domains}

We focus on the construction of an optimal tube connecting the initial domain $\Omega_{0}$ to the final domain $\Omega_{1}$, this optimal tube is also called a geodesic tube. The question is to determine, through the use of shape metrics $d\left(\Omega_{0}, \Omega_{1}\right)$ and $d_{h}\left(\Omega_{0}, \Omega_{1}\right)$, which tube is an optimal tube among all those tubes in the admissible set (see Fig. 3).

Let us consider the metrics $d$ and $d_{h}$ defined by (4) and (20) that we can rewrite as follows:

$$
\begin{aligned}
d\left(\Omega_{0}, \Omega_{1}\right) & =\inf _{\Phi \in \mathscr{T}_{L S}\left(\Omega_{0}, \Omega_{1}\right)} \int_{0}^{1} J\left(\Gamma_{t}\right) d t \\
d_{h}\left(\Omega_{0}, \Omega_{1}\right) & =\inf _{\Phi \in \mathscr{T}_{L S}\left(\Omega_{0}, \Omega_{1}\right)} \int_{0}^{1} J_{h}\left(\Gamma_{t}\right) d t
\end{aligned}
$$




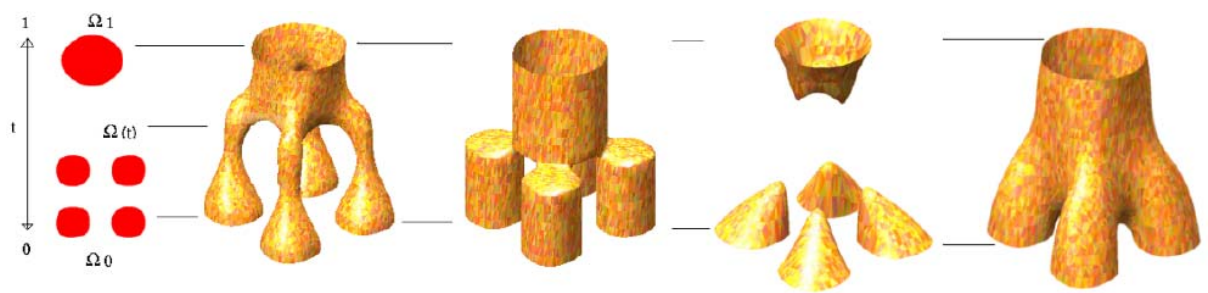

Fig. 3. Different continuous tubes between $\Omega_{0}$ and $\Omega_{1}$

where the functionals $J\left(\Gamma_{t}\right)$ and $J_{h}\left(\Gamma_{t}\right)$ are defined by:

$$
\begin{aligned}
J\left(\Gamma_{t}\right) & =\int_{\Gamma_{t}}|v(t, x)| d \Gamma(x)=\int_{\Gamma_{t}} \frac{\left|\partial_{t} \Phi(t, x)\right|}{\|\nabla \Phi(t, x)\|} d \Gamma(x) \\
J_{h}\left(\Gamma_{t}\right) & =\frac{1}{2 h} \int_{U_{h}\left(\Gamma_{t}\right)}\left|\partial_{t} \Phi(t, x)\right| d x
\end{aligned}
$$

Then, in order to solve the problem concerning the geodesic tube, that is to say to compute the metrics $d$ or $d_{h}$ defined by [44), we use a gradient method based on the computation of the shape derivative.

Lemma 5. According to (28), the eulerian derivative of the functional $J$ in the direction of a perturbation vector field $\mathbf{W} \in C_{0}^{1}(\mathbb{D} ; \mathbb{D})$ is:

$$
\begin{aligned}
d J\left(\Gamma_{t}, \mathbf{W}\right) & =\left.\int_{\Gamma_{t}} \partial_{\varepsilon}|v(t+\varepsilon, x)|\right|_{\varepsilon=0} d \Gamma(x) \\
& +\int_{\Gamma_{t}}\left[\frac{\partial|v(t, x)|}{\partial n}+H(t, x)|v(t, x)|\right]\langle\mathbf{W}(t, x), \mathbf{n}(t, x)\rangle_{\mathbb{R}^{n}} d \Gamma(x)
\end{aligned}
$$

where $H$ is the mean curvature. Using the level set formulation the eulerian derivative of the functional $J$ turns into:

$$
\begin{aligned}
d J\left(\Gamma_{t}, \mathbf{W}\right)= & \int_{\Gamma_{t}}\left[\left.\frac{\operatorname{sign}\left(\partial_{t} \Phi\right)}{\|\nabla \Phi\|} \partial_{\varepsilon}\left(\partial_{t} \Phi\right)\right|_{\varepsilon=0}-\left.\left|\partial_{t} \Phi\right| \frac{1}{\|\nabla \Phi\|^{3}} \nabla \Phi \cdot \nabla\left(\partial_{\varepsilon} \Phi\right)\right|_{\varepsilon=0}\right. \\
& +\left(-\operatorname{sign}\left(\partial_{t} \Phi\right) \frac{\nabla \Phi}{\|\nabla \Phi\|} \cdot \frac{\nabla\left(\partial_{t} \Phi\right)}{\|\nabla \Phi\|}+2\left|\partial_{t} \Phi\right| \frac{\nabla \Phi}{\|\nabla \Phi\|} \cdot\left[\frac{D^{2} \Phi}{\|\nabla \Phi\|^{2}} \cdot \frac{\nabla \Phi}{\|\nabla \Phi\|}\right]-\right. \\
& \left.\left.-\frac{\left|\partial_{t} \Phi\right|}{\|\nabla \Phi\|^{2}} \Delta \Phi\right) \frac{\partial_{\varepsilon} \Phi}{\|\nabla \Phi\|}\right] d \Gamma(x)
\end{aligned}
$$

Proof. According to the equation (46), the eulerian derivative of the functional $J_{l s}$ in the direction of a perturbation vector field $\mathbf{W}$ turns into: 


$$
\begin{aligned}
\left.\partial_{\varepsilon}|v(t+\varepsilon, x)|\right|_{\varepsilon=0} & =\left.\partial_{\varepsilon}\left(\frac{\left|\partial_{t} \Phi\right|}{\|\nabla \Phi\|}\right)\right|_{\varepsilon=0} \\
& =\left.\frac{\operatorname{sign}\left(\partial_{t} \Phi\right)}{\|\nabla \Phi\|} \partial_{\varepsilon}\left(\partial_{t} \Phi\right)\right|_{\varepsilon=0}+\left.\partial_{\varepsilon}\left(\frac{1}{\|\nabla \Phi\|}\right)\right|_{\varepsilon=0}\left|\partial_{t} \Phi\right| \\
& =\left.\frac{\operatorname{sign}\left(\partial_{t} \Phi\right)}{\|\nabla \Phi\|} \partial_{\varepsilon}\left(\partial_{t} \Phi\right)\right|_{\varepsilon=0}-\left.\left|\partial_{t} \Phi\right| \frac{1}{\|\nabla \Phi\|^{3}} \nabla \Phi \cdot \nabla\left(\partial_{\varepsilon} \Phi\right)\right|_{\varepsilon=0}
\end{aligned}
$$

and

$$
\begin{aligned}
& \frac{\partial|v(t, x)|}{\partial n}+H|v(t, x)|=\frac{-\nabla \Phi}{\|\nabla \Phi\|} \cdot \nabla\left(\frac{\left|\partial_{t} \Phi\right|}{\|\nabla \Phi\|}\right)+\nabla \cdot\left(\frac{-\nabla \Phi}{\|\nabla \Phi\|}\right) \frac{\left|\partial_{t} \Phi\right|}{\|\nabla \Phi\|} \\
& \frac{-\nabla \Phi}{\|\nabla \Phi\|} \cdot \nabla\left(\frac{\left|\partial_{t} \Phi\right|}{\|\nabla \Phi\|}\right)+\left[-\nabla\left(\frac{1}{\|\nabla \Phi\|}\right) \cdot \nabla \Phi-\frac{\Delta \Phi}{\|\nabla \Phi\|}\right] \frac{\left|\partial_{t} \Phi\right|}{\|\nabla \Phi\|} \\
& =\frac{-\nabla \Phi}{\|\nabla \Phi\|} \cdot \frac{\nabla\left(\left|\partial_{t} \Phi\right|\right)}{\|\nabla \Phi\|}-2\left|\partial_{t} \Phi\right| \frac{\nabla \Phi}{\|\nabla \Phi\|} \cdot \nabla\left(\frac{1}{\|\nabla \Phi\|}\right)-\frac{\left|\partial_{t} \Phi\right|}{\|\nabla \Phi\|^{2}} \Delta \Phi \\
& =-\operatorname{sign}\left(\partial_{t} \Phi\right) \frac{\nabla \Phi}{\|\nabla \Phi\|} \cdot \frac{\nabla\left(\partial_{t} \Phi\right)}{\|\nabla \Phi\|}+ \\
& +2\left|\partial_{t} \Phi\right| \frac{\nabla \Phi}{\|\nabla \Phi\|} \cdot\left[\frac{D^{2} \Phi}{\|\nabla \Phi\|^{2}} \cdot \frac{\nabla \Phi}{\|\nabla \Phi\|}\right]-\frac{\left|\partial_{t} \Phi\right|}{\|\nabla \Phi\|^{2}} \Delta \Phi
\end{aligned}
$$

Lemma 6. According to (28), the eulerian derivative of the functional $J_{h}$ in the direction of a perturbation vector field $\mathbf{W} \in C_{0}^{1}(\mathbb{D} ; \mathbb{D})$ is:

$$
\begin{aligned}
d J_{h}\left(\Gamma_{t}, \mathbf{W}\right) & =\frac{1}{2 h} \int_{\mathbb{D}}\left[\left.\partial_{\varepsilon}\left(\left|\partial_{t} \Phi(t+\varepsilon, x)\right|\right)\right|_{\varepsilon=0} \rho_{h} \circ b_{\Omega_{t}}(x)\right] d x \\
& +\frac{1}{2 h} \int_{\mathbb{D}}\left[\left.\left|\partial_{t} \Phi(t, x)\right| \partial_{\varepsilon}\left(\rho_{h} \circ b_{\Omega_{t+\varepsilon}}(x)\right)\right|_{\varepsilon=0}\right] d x
\end{aligned}
$$

where the function $\rho_{h}$ is defined by: $\rho_{h}(x)=\left\{\begin{array}{ll}\frac{x}{h}+1 & \text { if } x \in[-h, 0] \\ \frac{-x}{h}+1 & \text { if } x \in[0, h] \\ 0 & \text { if } x \in \mathbb{R} \backslash[-h, h]\end{array}\right.$.

Proof. Due to the fact that $\left.\rho_{h} \circ b_{\Omega_{t}}(x)\right|_{\Gamma_{t}}=1$, and using the fact that $\operatorname{supp}\left(\rho_{h} \circ b_{\Omega_{t}}\right) \subseteq$ $U_{h}\left(\Gamma_{t}\right)$ we can rewrite the functional $J_{h}$ as follows

$$
J_{h}\left(\Gamma_{t}\right)=\frac{1}{2 h} \int_{\mathbb{D}}\left|\partial_{t} \Phi(t, x)\right| \rho_{h} \circ b_{\Omega_{t}}(x) d x
$$

Consequently, the eulerian derivative of the functional $J_{h}$ turns into the equation 51 where:

$$
\left.\partial_{\varepsilon}\left(\left|\partial_{t} \Phi(t+\varepsilon, x)\right|\right)\right|_{\varepsilon=0}=\left.\operatorname{sign}\left(\partial_{t} \Phi\right) \partial_{\varepsilon}\left(\partial_{t} \Phi(t, x)\right)\right|_{\varepsilon=0} .
$$


Using $\partial_{\varepsilon}\left(b_{\Omega_{t}}(x)\right)+\nabla b_{\Omega_{t}}(x)$. Wop $=0$ and $\nabla b_{\Omega_{t}}(x)=\mathbf{n}(t, x)$, we get

$$
\begin{aligned}
\left.\partial_{\varepsilon}\left(\rho_{h} \circ b_{\Omega_{t}}(x)\right)\right|_{\varepsilon=0} & =\rho_{h}^{\prime} \circ b_{\Omega_{t}}(x) \partial_{\varepsilon}\left(b_{\Omega_{t}}(x)\right) \\
& =-\rho_{h}^{\prime} \circ b_{\Omega_{t}}(x)\langle\mathbf{W}, \mathbf{n}\rangle_{\mathbb{R}^{n}} \\
& =-\rho_{h}^{\prime} \circ b_{\Omega_{t}}(x) \frac{\partial_{\varepsilon} \Phi}{\|\nabla \Phi\|}
\end{aligned}
$$

The derivative of the function $\rho_{h}(x)$ is defined by:

$$
\rho_{h}^{\prime}(x)=\left\{\begin{array}{ll}
\frac{1}{h} & \text { if } x \in[-h, 0] \\
\frac{-1}{h} & \text { if } x \in[0, h] \\
0 & \text { if } x \in \mathbb{R} \backslash[-h, h]
\end{array}= \begin{cases}\frac{1}{h}\left(1-2 \chi_{\Omega_{t}}(x)\right) & \text { if } x \in U_{h}\left(\Gamma_{t}\right) \\
0 & \text { if } x \in \mathbb{R} \backslash[-h, h]\end{cases}\right.
$$

Finally, we get for the eulerian derivative of the functional $J_{h}$ in the direction of a perturbation vector field $\mathbf{W} \in C_{0}^{1}(\mathbb{D} ; \mathbb{D})$ :

$$
\begin{aligned}
d J_{h}\left(\Gamma_{t}, \mathbf{W}\right) & =\frac{1}{2 h} \int_{\mathbb{D}}\left[\left.\operatorname{sigh}\left(\partial_{t} \Phi\right) \partial_{\varepsilon}\left(\partial_{t} \Phi(t, x)\right)\right|_{\varepsilon=0} \rho_{h} \circ b_{\Omega_{t}}(x)\right] d x \\
& -\frac{1}{2 h} \int_{\mathbb{D}}\left[\left|\partial_{t} \Phi(t, x)\right| \rho_{h}^{\prime} \circ b_{\Omega_{t}}(x) \frac{\partial_{\varepsilon} \Phi}{\|\nabla \Phi\|}\right] d x
\end{aligned}
$$

\subsubsection{Polynomial Decomposition of the Parameter $\Lambda(t)$}

We continue the study of a geodesic tube through a tube formulation using a GalerkinLevel set strategy. Consequently, $\Lambda(t) \in \mathscr{T}_{\Lambda}\left(\Omega_{0}, \Omega_{1}\right)$ represents the parameters of the optimization process. For complexity reason, we consider a polynomial decomposition of the parameter $\Lambda(t)$ as follows:

$$
\Lambda(t)=P_{\alpha}(t) \Lambda_{1}+\left(1-P_{\alpha}(t)\right) \Lambda_{0}, \quad P_{\alpha}(t)=\sum_{i=1}^{M} \alpha_{i} e_{i}(t)
$$

where $\alpha=\left(\alpha_{1}, \ldots, \alpha_{M}\right)$ are the coefficients of the decomposition of the polynomial $P_{\alpha}(t)$ in the basis $\left\{e_{1}(t), \ldots, e_{M}(t)\right\}$. Consequently, the feasible set of connecting tubes defined by (43) with initial and final conditions on $\Lambda(t)$ turns into a feasible set with initial and final conditions on the polynomial $P_{\alpha}$ defined as follows:

$$
\mathscr{T}_{\alpha}\left(\Omega_{0}, \Omega_{1}\right)=\left\{\alpha \in \mathbb{R}^{M},\left[\begin{array}{l}
P_{\alpha}(0)=0 \\
P_{\alpha}(1)=1
\end{array}\right\}\right.
$$

Let us consider the metrics $d$ and $d_{h}$ defined by (4) and (20) that we can rewrite as follows:

$$
\begin{aligned}
d\left(\Omega_{0}, \Omega_{1}\right) & =\inf _{\alpha \in \mathscr{T}_{\alpha}\left(\Omega_{0}, \Omega_{1}\right)} \int_{0}^{1} \tilde{J}\left(\Gamma_{t}\right) d t \\
d_{h}\left(\Omega_{0}, \Omega_{1}\right) & =\inf _{\alpha \in \mathscr{T}_{\alpha}\left(\Omega_{0}, \Omega_{1}\right)} \int_{0}^{1} \tilde{J_{h}}\left(\Gamma_{t}\right) d t
\end{aligned}
$$


where the functionals $\tilde{J}\left(\Gamma_{t}\right)$ and $\tilde{J}_{h}\left(\Gamma_{t}\right)$ are defined by:

$$
\begin{aligned}
\tilde{J}\left(\Gamma_{t}\right) & =\left|\dot{P}_{\alpha}(t)\right| \int_{\Gamma_{t}} \frac{\left|\Phi_{1}(x)-\Phi_{0}(x)\right|}{\|\nabla \Phi\|} d \Gamma(x) \\
\tilde{J}_{h}\left(\Gamma_{t}\right) & =\frac{\left|\dot{P}_{\alpha}(t)\right|}{2 h} \int_{U_{h}\left(\Gamma_{t}\right)}\left|\Phi_{1}(x)-\Phi_{0}(x)\right| d x
\end{aligned}
$$

Then, in order to solve the problem concerning the geodesic tube, that is to say to compute the metrics $d$ or $d_{h}$ defined by (58), we use a gradient method based on the computation of the shape derivative.

Assumption 2. The shape derivative of the functional J defined by (46) can be rewritten as follows:

$$
d J\left(\Gamma_{t}, \mathbf{W}\right)=\left.\frac{\partial J(\alpha+\varepsilon h)}{\partial \varepsilon}\right|_{\varepsilon=0}=\left\langle h, \nabla J\left(\Gamma_{t}\right)\right\rangle_{\mathbb{R}^{M}}
$$

where $\forall i \in[1, M]$ :

$$
\begin{aligned}
\left(\nabla J\left(\Gamma_{t}\right)\right)_{i}= & \dot{e}_{i}(t) \operatorname{sign}\left(\dot{P}_{\alpha}(t)\right) \int_{\Gamma_{t}} \frac{\left|\Phi_{1}(x)-\Phi_{0}(x)\right|}{\|\nabla \Phi\|} d \Gamma(x) \\
& +e_{i}(t)\left|\dot{P}_{\alpha}(t)\right|, \int_{\Gamma_{t}} \frac{\left|\Phi_{1}(x)-\Phi_{0}(x)\right|}{\|\nabla \Phi\|} K(t, x) d \Gamma(x)
\end{aligned}
$$

and

$$
\begin{aligned}
& K(t, x)=\left[-2 \frac{\nabla \Phi \cdot\left(\nabla \Phi_{1}(x)-\nabla \Phi_{0}(x)\right)}{\|\nabla \Phi\|^{2}}+\right. \\
& +2\left(\Phi_{1}(x)-\Phi_{0}(x)\right) \frac{\nabla \Phi}{\|\nabla \Phi\|} \cdot\left[\frac{D^{2} \Phi}{\|\nabla \Phi\|^{2}} \cdot \frac{\nabla \Phi}{\|\nabla \Phi\|}\right] \\
& \left.-\left(\Phi_{1}(x)-\Phi_{0}(x)\right) \frac{\Delta \Phi}{\|\nabla \Phi\|^{2}}\right]
\end{aligned}
$$

Assumption 3. The shape derivative of the functional $J_{h}$ defined by (51) can be rewritten as follows:

$$
d J_{h}\left(\Gamma_{t}, \mathbf{W}\right)=\left.\frac{\partial J_{h}(\alpha+\varepsilon h)}{\partial \varepsilon}\right|_{\varepsilon=0}=\left\langle h, \nabla J_{h}\left(\Gamma_{t}\right)\right\rangle_{\mathbb{R}^{M}}
$$

where $\forall i \in[1, M]$ :

$$
\begin{aligned}
&\left(\nabla J_{h}\left(\Gamma_{t}\right)\right)_{i}= \frac{\dot{e}_{i}(t)}{2 h} \operatorname{sign}\left(\dot{P}_{\alpha}(t)\right) \int_{U_{h}\left(\Gamma_{t}\right)}\left|\Phi_{1}(x)-\Phi_{0}(x)\right| d x \\
&-\frac{e_{i}(t)}{2 h^{2}}\left|\dot{P}_{\alpha}(t)\right| \int_{U_{h}\left(\Gamma_{t}\right)}\left(1-2 \chi_{\Omega_{t}}(x)\right) \mid \Phi_{1}(x)- \\
& \Phi_{0}(x) \mid \frac{\left(\Phi_{1}(x)-\Phi_{0}(x)\right)}{\|\nabla \Phi\|} d x
\end{aligned}
$$




\section{Algorithm 2}

I. Initialization: Choose an initial vector of parameters $\Lambda(t)$ defined by (56) which generate an admissible connecting tube between $\Omega_{0}$ and $\Omega_{1}$ through the choice of the parameter $\alpha$. Initialize the level set function $\Phi(t, x)=P_{\alpha}(t) \Phi_{1}(x)+\left(1-P_{\alpha}(t)\right) \Phi_{0}(x)$. Set $k=0$.

II. Shape gradient direction: For every $t \in I$, find the tubular neighborhood $U_{h}\left(\Gamma_{t}\right)$ of the zero level set $\Gamma_{t}$ of the actual level set function $\Phi(t, x)$. Compute $\nabla J_{h}\left(\Gamma_{t}\right)$ from the equation (64).

\section{Update:}

- Perform a time step to update $\alpha$.

- Let $\alpha^{+}$denote this update: $\alpha^{+}=\alpha-\rho \int_{0}^{1} \nabla J_{h}\left(\Gamma_{t}\right) d t, \rho>0$.

- Update the function $\Phi^{+}(t, x)=P_{\alpha^{+}}(t) \Phi_{1}(x)+\left(1-P_{\alpha^{+}}(t)\right) \Phi_{0}(x)$.

- Set $k=k+1$ and go to (2).
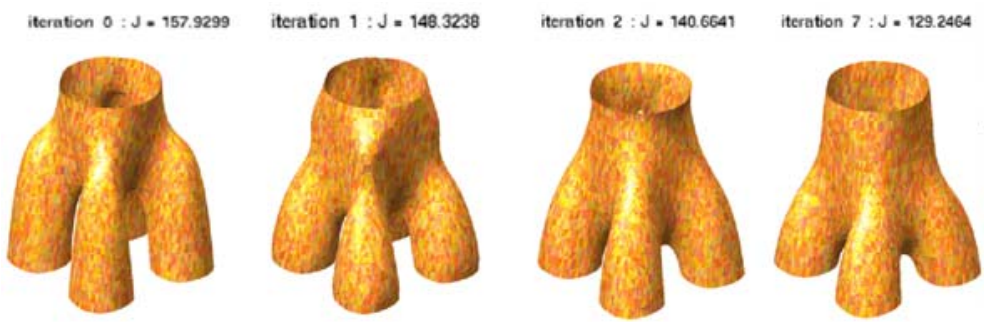

Fig. 4. Tube optimization using the metric $d_{h}\left(\Omega_{0}, \Omega_{1}\right)$

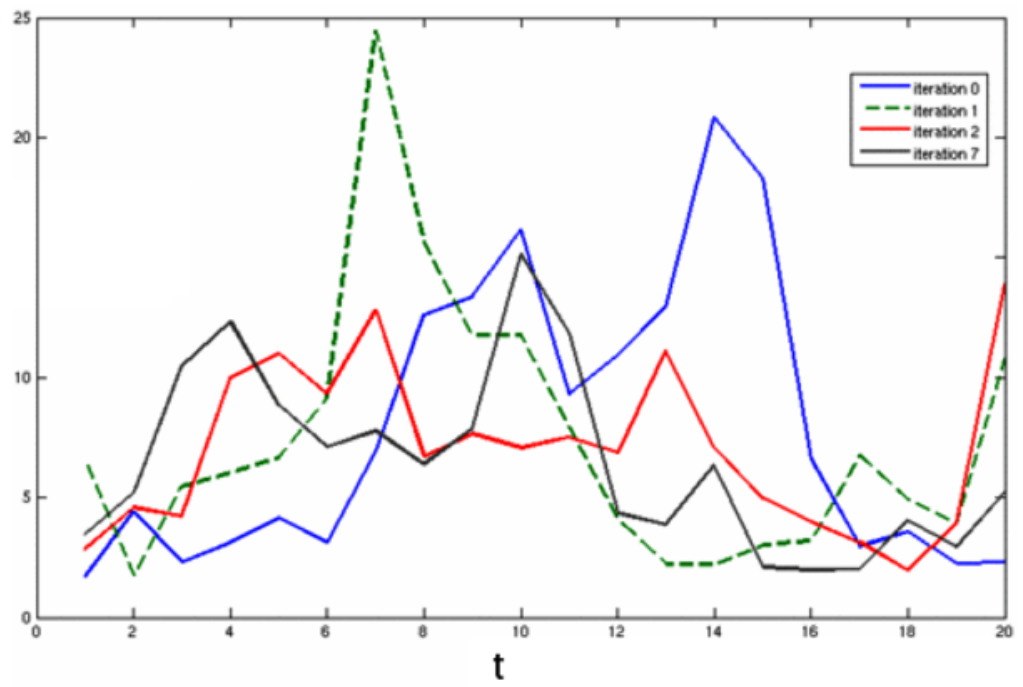

Fig. 5. Distribution of the functional values $J_{h}\left(\Gamma_{t}\right)$ for tube obtained during the optimization process of Fig. 4 


\subsection{Numerical Experiment of a Geodesic Tube Construction}

We present a numerical experiment based on the algorithm 2 for a 3D tube optimization. Fig. (4) shows tubes obtained during the optimization process for different iterations. From Fig. 5 we can see that the tube obtained after seven iterations has a more homogeneous distribution of the functional values $J_{h}\left(\Gamma_{t}\right)$ compared to the initial tube. The result of this optimization process is the construction of a smoother tube than the initial tube.

\section{References}

1. Blanchard, L., Zolésio, J.-P.: Morphing by moving. Shape modeling with Galerkin approximation. In: Sivasundaram, S. (ed.) International Conference on Nonlinear Problems in Aviation and Aerospace (to appear)

2. Delfour, M.C., Zolésio, J.-P.: Oriented distance functions in shape analysis and optimization. In: Control and Optimal Design of Distributed Parameter Systems. Mathematics and its Applications, pp. 39-72. Springer, Heidelberg (1995)

3. Delfour, M.C., Zolésio, J.P.: Shapes and Geometries. Analysis, Differential Calculus, and Optimization. Advances in Design and Control. SIAM, Philadelphia (2001)

4. Zolésio, J.-P.: Set Weak Evolution and Transverse Field, Variational Application and Shape Differential Equation. INRIA report RR-4649 (2002), http: //www.inria.fr/rrrt/rr-4649.html

5. Zolésio, J.-P.: Shape Topology by Tube Geodesic. In: Information Processing: Recent Mathematical Advances in Optimization and Control, pp. 185-204. Presse de l'Ecole des Mines de Paris (2004)

6. Zolésio, J.-P.: Optimal tubes: geodesic metric, Euler flow, moving domain. In: Free and Moving Boundaries: Analysis, Simulation and Control, vol. 252, pp. 203-213. Taylor \& Francis, CRC Press (2005) 